\title{
DETERMINAÇÃO ESPECTROSCÓPICA MULTIVARIADA DE GLUCOSAMINA E CONDROITINA EM FORMULAÇÕES FARMACÊUTICAS
}

\author{
Paula Rossignoli, Roberto Pontarolo* e Cassyano J. Correr
}

Departamento de Farmácia, Universidade Federal do Paraná, Av. Prefeito Lothário Meissner, 632, 80210-170 Curitiba - PR, Brasil

Gilcelia Cordeiro e Patricio Peralta-Zamora

Departamento de Química, Universidade Federal do Paraná, CP 19081, 81531-990 Curitiba - PR, Brasil

Recebido em 29/1/07; aceito em 11/1/08; publicado na web em 31/7/08

\begin{abstract}
MULTIVARIATE SPECTROSCOPIC DETERMINATION OF GLUCOSAMINE AND CHONDROITIN IN PHARMACEUTICAL FORMULATIONS. The objective of this study was to develop and validate an analytical method for quantification of glucosamine and chondroitin in pharmaceutical formulations. Multivariate calibration combined with infrared spectrophotometry allowed this analysis. 25 mixtures of glucosamine-6-sulphate and chondroitin-6-sulphate were used for calibration. Average errors found with this model during external validation were $1.37 \%$ for glucosamine sulphate and $1.30 \%$ for chondroitin sulphate. This method presented satisfactory results for assessed variables, what indicating that it is suitable for simultaneous quantification of glucosamine and chondroitin.
\end{abstract}

Keywords: glucosamine; chondroitin; analytical method validation.

\section{INTRODUÇÃO}

Glucosamina e condroitina são substâncias de ocorrência natural, componentes da cartilagem articular. A glucosamina é um aminoaçúcar e está envolvida na formação da cartilagem, atuando como precursora de unidades de dissacarídeos dos glicosaminoglicanos. A condroitina é um glicosaminoglicano, cujas unidades de dissacarídeos contêm um aminoaçúcar (galactosamina) e participa da formação de proteoglicanos. ${ }^{1}$

A glucosamina-6-sulfato e a condroitina-6-sulfato (Figura 1) são substâncias que vêm sendo utilizadas, em associação, no tratamento da osteoartrite, com doses diárias recomendadas de 1500 e 1200 mg, respectivamente. ${ }^{2}$

Ambas as substâncias não apresentam absorção característica na região do ultravioleta-visível (200 a $700 \mathrm{~nm}$ ), enquanto que um perfil de absorção bastante semelhante é verificado na região do infravermelho. Tratam-se de características que limitam a aplicabilidade de metodologias espectroscópicas fundamentadas em processos convencionais de calibração.

Nos últimos anos, os avanços na área da quimiometria e a massificação dos recursos computacionais têm propiciado o desenvolvimento

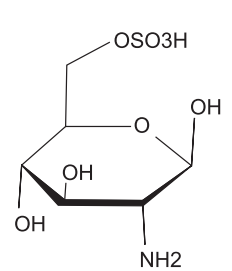

A

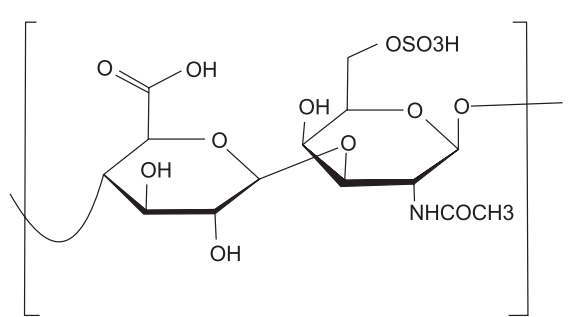

B
Figura 1. Estrutura química da glucosamina-6-sulfato (A) e da condroitina6-sulfato (B)

\footnotetext{
*e-mail: pontarolo@ufpr.br
}

de métodos de calibração multivariada, os quais permitem a quantificação de substâncias ativas em associação, ${ }^{3}$ mesmo em condições de interferência ou complexidade espectral.

Várias publicações têm ilustrado a utilidade dos processos de calibração multivariada na determinação espectroscópica simultânea de princípios ativos de interesse em formulações farmacêuticas de relevância ${ }^{4,5}$ e também de princípios ativos de uso tópico presentes em matrizes como cremes e pomadas. ${ }^{3}$ Adicionalmente, técnicas que recorrem a modelos multivariados associados à espectroscopia no infravermelho têm sido utilizadas com sucesso na análise de polissacarídeos. ${ }^{6}$

A espectroscopia no infravermelho com reflectância difusa (DRIFTS - Diffuse Reflection Infrared Fourier Transform Spectroscopy $)^{7}$ associada à calibração multivariada revela-se um método analítico que possibilita a quantificação simultânea de glucosamina e condroitina em forma farmacêutica. Tal técnica tem sido utilizada com frequiência pela indústria farmacêutica ${ }^{8,9} \mathrm{e}$ tem se tornado padrão para análises de amostras sólidas pulverizadas, ${ }^{10}$ permitindo a rápida aquisição de dados espectrais sem significativa alteração da sua forma original. ${ }^{11,12}$

O processo de calibração multivariada na análise de dados espectroscópicos tem sido bastante utilizado em análises farmacêuticas, principalmente para amostras compostas por múltiplos componentes ou interferentes, partindo-se de dados instrumentais de baixa seletividade. ${ }^{13}$ Entretanto, a aceitação e a implementação deste método têm sofrido restrições quanto à ausência de validação de acordo com os critérios dos órgãos de fiscalização nacionais e internacionais, como ANVISA (Agência Nacional de Vigilância Sanitária) e ICH (International Conference on Harmonisation's). ${ }^{14}$

Este trabalho apresenta o desenvolvimento e validação de um método para determinação simultânea de glucosamina e condroitina em formulações farmacêuticas (cápsulas), recorrendo à espectroscopia no infravermelho e à regressão de mínimos quadrados parciais (PLSR). 


\section{PARTE EXPERIMENTAL}

\section{Amostras e padrões}

Para o preparo dos conjuntos de calibração e validação foram utilizados padrões Sigma ${ }^{\circledR}$ de glucosamina-6-sulfato, condroitina-6sulfato e estearato de magnésio. Amostras comerciais, na forma de cápsulas, foram obtidas de diferentes farmácias de manipulação, nas concentrações de $400 \mathrm{mg}$ de condroitina e $500 \mathrm{mg}$ de glucosamina.

\section{Desenvolvimento do modelo de calibração}

Para a construção do modelo de calibração foram preparadas 25 misturas sintéticas, contendo entre 400 e $600 \mathrm{mg}$ de glucosamina-6-sulfato, entre 320 e $480 \mathrm{mg}$ de condroitina-6-sulfato e entre 0 a $140 \mathrm{mg}$ de estearato de magnésio, por grama de mistura. Como diluente foi escolhido estearato de magnésio, em razão de ser o excipiente presente nas formulações em cápsula. As misturas foram preparadas utilizando-se sempre a mesma ordem de adição das substâncias e o mesmo procedimento e tempo de trituração e homogeneização.

Para a validação externa do modelo foram preparadas 5 misturas, cujas concentrações estavam dentro do intervalo estabelecido para as amostras de calibração, mas que não foram utilizadas no conjunto de calibração.

Os espectros na região do infravermelho foram adquiridos entre 7000 e $400 \mathrm{~cm}^{-1}$, utilizando-se espectrofotômetro Excalibur (modelo FTS-4000 Bio-Rad) e acessório de reflectância difusa.

A região espectral utilizada compreendeu os intervalos de 6400 a 6200,5950 a 5700,5300 a 3800 e 1600 a $1500 \mathrm{~cm}^{-1}$, correspondendo a 910 números de onda no infravermelho próximo e 156 números de onda no infravermelho médio. A escolha da região espectral para a construção do modelo foi baseada na comparação do espectro das substâncias puras com o espectro das misturas, selecionando-se regiões de boa reprodutibilidade.

Para a construção das matrizes de dados foi utilizado o software Origin Pro $6.1^{\circledR}$, enquanto que para a construção dos modelos o programa PLS-toolbox 1.5, que opera em ambiente Matlab v.4.2. Durante o desenvolvimento dos modelos, os dados das matrizes $\mathrm{X}$ e $\mathrm{Y}$ foram centrados na média e, além desse, outros pré-processamentos como suavização de sinais analíticos com filtro de Savitzky-Golay, seguido de derivação e correção do fator multiplicativo do sinal (MSC), foram realizados.

\section{Validação do método}

O método desenvolvido foi avaliado em relação a parâmetros de precisão (repetitividade e precisão intermediária) e exatidão. Para a avaliação desses parâmetros foram preparadas triplicatas de 4 misturas com diferentes concentrações de padrões de glucosamina e de condroitina dentro do intervalo de concentração estabelecido no conjunto de calibração. A validação seguiu as normas vigentes da Resolução n 899 de 29 de maio de 2003 da Agência Nacional de Vigilância Sanitária. ${ }^{15}$

\section{Análise de medicamentos}

Após o desenvolvimento e validação do método analítico, 3 formulações comerciais contendo a associação em estudo (glucosamina sulfato: $500 \mathrm{mg}$ e condroitina sulfato: $400 \mathrm{mg}$ ) foram obtidas em farmácias de manipulação de Curitiba-PR, tendo a sua composição determinada pelo método proposto.

\section{RESULTADOS E DISCUSSÃO}

\section{Caracterização espectral dos fármacos}

Os espectros de reflexão difusa no infravermelho da glucosamina6-sulfato da condroitina-6-sulfato e da mistura sintética das duas substâncias são apresentados na Figura 2.

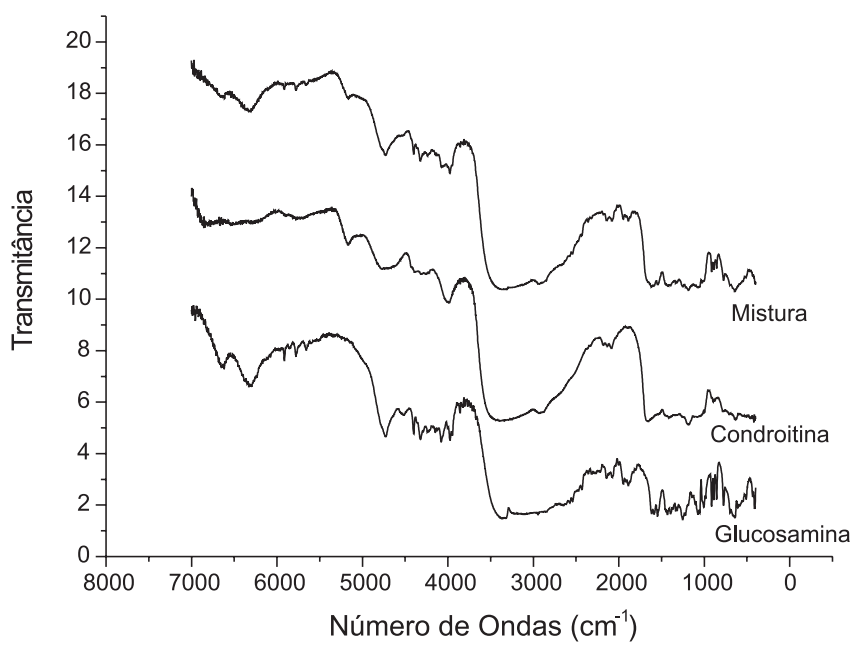

Figura 2. Espectro de infravermelho com reflectância difusa (DRIFTS) de glucosamina-6-sulfato e condroitina-6-sulfato

Glucosamina-6-sulfato e condroitina-6-sulfato diferem quanto aos grupos químicos presentes e ao tipo de monossacarídeo. Condroitina-6-sulfato é um glicosaminoglicano constituído de resíduos alternados de ácido urônico e $\mathrm{N}$-acetil- $D$-galactosamina-6-sulfato, sendo que as unidades de dissacarídeo estão unidas umas às outras através de ligação $\beta 1-4$ e os resíduos estão unidos uns aos outros através de ligação $\beta 1-3$, apresentando, portanto, na sua estrutura grupamento carboxílico, $\mathrm{N}$-acetil e sulfato, além de ligações glicosídicas. A glucosamina-6-sulfato é um monossacarídeo que contém um grupo amino e um grupo sulfato.

Essas diferenças na estrutura química são responsáveis pelas diferenças observadas nos espectros das substâncias puras e podem influenciar a seletividade do método.

\section{Desenvolvimento dos modelos multivariados}

Na etapa de calibração, foi verificado que o somatório dos erros de previsão (PRESS), calculado conforme a Equação 1, diminui significativamente com a introdução de um maior número de variáveis latentes (VLs), até o limite de 8 VLs. A partir deste ponto, a introdução de novas VLs não melhora significativamente a capacidade de previsão, ao mesmo tempo em que não se produzem ganhos significativos em termos de representação da variância de Y (concentração das espécies em estudo). Adicionalmente, o uso de 8 variáveis latentes e qualquer tipo de pré-processamento implica a representação de aproximadamente $99 \%$ da variância de X (informações espectrais).

$\operatorname{PRESS}=\Sigma\left(\mathrm{y}^{\prime}-\mathrm{y}\right)^{2}$

onde y'é o valor conhecido e y é o valor calculado.

De maneira geral, trabalhar com um número de VLs que representa mais que $99 \%$ da variância dos dados espectrais leva à obtenção de modelos de baixa robustez, em razão de levar em consideração até as pequenas flutuações instrumentais. 
Sendo assim, modelos foram desenvolvidos com 2 a 8 variáveis latentes, utilizando-se, concomitantemente, diversos tipos de préprocessamento. Dentre os vários sistemas avaliados, destaque pode ser dado aos dados centrados na média (pré-processamento 1), à suavização de sinais analíticos com filtro de Savitzky-Golay (janela otimizada de 15 pontos e polinômio de segunda ordem) seguido de derivação (pré-processamento 2) e à correção do fator multiplicativo do sinal (MSC) (pré-processamento 3), este último especialmente útil no tratamento de dados espectrais obtidos no modo de refletância.

A seleção do melhor modelo de previsão foi fundamentada na avaliação do erro relativo médio de validação, obtido na análise das 5 amostras reservadas para este fim.

Os resultados, apresentados na Figura 3, indicam que o sistema de alisamento e derivação proporciona os maiores erros de previsão (7,7 a 9,0 para glucosamina e 9,8 a 12,2 para condroitina), resultados que não melhoram com a introdução de um maior número de VLs. O alisamento e cálculo da derivada são utilizados para corrigir variações na linha de base e reduzir o efeito de ruídos instrumentais. Neste caso, a obtenção de resultados incoerentes evidencia a pouca seletividade dos filtros de suavização, fato que normalmente redunda na remoção simultânea de ruídos e sinais analíticos de relevância. ${ }^{16}$

Por sua vez, a utilização de dados centrados na média e corrigidos pelo fator multiplicativo de sinal (MSC) proporciona modelos com excelente capacidade de previsão, com erros relativos médios entre 2 e $4 \%$, para qualquer número de VLs utilizadas.

A melhor capacidade de previsão foi proporcionada pelo modelo que opera com 5 VLs e pré-processamento por MSC. Nestas condições, o erro médio de previsão é de 1,4\% para glucosamina e de $1,5 \%$ para condroitina. Em função deste fato, este modelo foi selecionado para a realização de estudos de validação.

\section{Validação do método}

\section{Repetitividade e precisão intermediária}

Neste estudo, 4 amostras de diferentes concentrações foram analisadas. Cada uma delas foi preparada e medida em triplicata, gerando 12 resultados para cada concentração. Na Tabela 1 são apresentadas as médias de previsão, a média final de previsão obtida, o desvio padrão e o coeficiente de variação para o ensaio de repetitividade.

As análises para o ensaio de precisão intermediária foram realizados por dois analistas em dias diferentes com intervalo de 7 dias

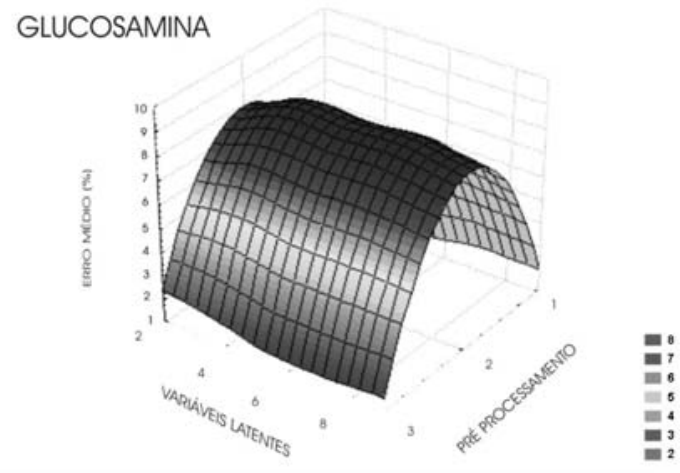

A

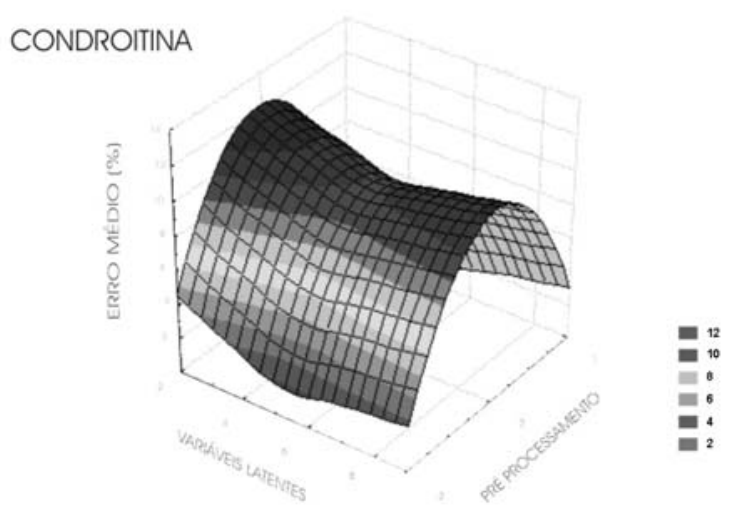

B

Figura 3. Efeito do número de variáveis latentes e do tipo de préprocessamento no erro médio de previsão, na fase de validação externa, para glucosamina (A) e condroitina (B)

no mesmo laboratório e com o mesmo equipamento. Na Tabela 2 são apresentadas as médias de previsão, a média final de previsão obtida, o desvio padrão e o coeficiente de variação para o ensaio de precisão intermediária.

Os resultados do ensaio de repetitividade e de precisão interme-

Tabela 1. Resultado das determinações de glucosamina e condroitina no ensaio de repetitividade

\begin{tabular}{|c|c|c|c|c|c|c|c|c|}
\hline \multirow[t]{2}{*}{ Concentração } & \multicolumn{2}{|c|}{$\begin{array}{l}\text { Valores encontrados } \\
\qquad(\mathrm{mg} / \mathrm{g})\end{array}$} & \multicolumn{2}{|c|}{$\begin{array}{l}\text { Média } \\
(\mathrm{mg} / \mathrm{g})\end{array}$} & \multicolumn{2}{|c|}{ Desvio Padrão } & \multicolumn{2}{|c|}{$\begin{array}{l}\text { Coeficiente de } \\
\text { Variação (\%) }\end{array}$} \\
\hline & Gluco & Condro & Gluco & Condro & Gluco & Condro & Gluco & Condro \\
\hline 1 (Gluco $400 \mathrm{mg} / \mathrm{g}$ & 406,1 & 475,5 & & & & & & \\
\hline Condro $480 \mathrm{mg} / \mathrm{g}$ ) & $\begin{array}{l}393,2 \\
395,3\end{array}$ & $\begin{array}{l}488,3 \\
482,2\end{array}$ & 398,2 & 482,0 & 6,9 & 6,4 & 1,7 & 1,3 \\
\hline $\begin{array}{l}2(\text { Gluco } 500 \mathrm{mg} / \mathrm{g} \\
\text { Condro } 400 \mathrm{mg} / \mathrm{g} \text { ) }\end{array}$ & $\begin{array}{l}499,0 \\
510,7 \\
512,4\end{array}$ & $\begin{array}{l}408,9 \\
410,0 \\
397,5\end{array}$ & 507,3 & 405,5 & 7,3 & 6,9 & 1,4 & 1,71 \\
\hline $\begin{array}{l}3 \text { (Gluco } 400 \mathrm{mg} / \mathrm{g} \\
\text { Condro } 320 \mathrm{mg} / \mathrm{g} \text { ) }\end{array}$ & $\begin{array}{l}390,7 \\
388,8 \\
396,5\end{array}$ & $\begin{array}{l}322,3 \\
325,3 \\
319,6\end{array}$ & 392,0 & 322,4 & 4,0 & 2,9 & 1,0 & 0,9 \\
\hline $\begin{array}{l}4 \text { (Gluco } 600 \mathrm{mg} / \mathrm{g} \\
\text { Condro } 320 \mathrm{mg} / \mathrm{g} \text { ) }\end{array}$ & $\begin{array}{l}606,0 \\
595,2 \\
593,7\end{array}$ & $\begin{array}{l}316,0 \\
329,9 \\
324,9\end{array}$ & 598,3 & 323,6 & 6,7 & 7,0 & 1,1 & 2,2 \\
\hline
\end{tabular}


Tabela 2. Resultados da determinação de glucosamina e condroitina no ensaio de precisão intermediária

\begin{tabular}{|c|c|c|c|c|c|c|c|c|c|c|}
\hline \multirow[t]{2}{*}{ Concentração } & \multicolumn{2}{|c|}{$\begin{array}{l}\text { Determinações } \\
\text { Analista } 1(\mathrm{mg} / \mathrm{g})\end{array}$} & \multicolumn{2}{|c|}{$\begin{array}{c}\text { Determinações } \\
\text { Analista } 2(\mathrm{mg} / \mathrm{g})\end{array}$} & \multicolumn{2}{|c|}{ Média } & \multicolumn{2}{|c|}{$\begin{array}{l}\text { Desvio } \\
\text { Padrão }\end{array}$} & \multicolumn{2}{|c|}{$\begin{array}{c}\text { Coeficiente } \\
\text { de Variação (\%) }\end{array}$} \\
\hline & Gluco & Condro & Gluco & Condro & Gluco & Condro & Gluco & Condro & Gluco & Condro \\
\hline 1 (Gluco $400 \mathrm{mg} / \mathrm{g}$ & 406,1 & 475,5 & 410,7 & 485,3 & & & & & & \\
\hline \multirow[t]{2}{*}{ Condro 480 mg/g) } & 393,2 & 488,3 & 418,6 & 486,9 & 402,5 & 482,7 & 6,7 & 5,1 & 1,7 & 1,1 \\
\hline & 395,3 & 482,2 & 408,9 & 477,7 & & & & & & \\
\hline 2 (Gluco 500 mg/g & 499,0 & 408,9 & 518,3 & 394,3 & & & & & & \\
\hline \multirow[t]{2}{*}{ Condro 400 mg/g) } & 510,7 & 410,0 & 506,2 & 389,9 & 511,1 & 397,8 & 7,8 & 9,8 & 1,5 & 2,5 \\
\hline & 512,4 & 397,5 & 520,2 & 386,2 & & & & & & \\
\hline 3 (Gluco $400 \mathrm{mg} / \mathrm{g}$ & 390,7 & 322,3 & 412,8 & 315,9 & & & & & & \\
\hline \multirow[t]{2}{*}{ Condro 320 mg/g) } & 388,8 & 325,3 & 409,3 & 305,6 & 401,7 & 316,9 & 11,0 & 7,1 & 2,7 & 2,2 \\
\hline & 396,5 & 319,6 & 412,1 & 312,7 & & & & & & \\
\hline 4 (Gluco 600 mg/g & 606,0 & 316,0 & 610,7 & 312,2 & & & & & & \\
\hline \multirow[t]{2}{*}{ Condro 320 mg/g) } & 595,2 & 329,9 & 612,8 & 306,4 & 603,6 & 319,5 & 7,9 & 6,6 & 1,3 & 2,1 \\
\hline & 593,7 & 324,9 & 602,9 & 315,9 & & & & & & \\
\hline
\end{tabular}

Tabela 3. Resultados do teor de glucosamina e condroitina no ensaio de recuperação

\begin{tabular}{|c|c|c|c|c|c|c|c|c|}
\hline \multirow[t]{2}{*}{ Concentração } & \multicolumn{2}{|c|}{$\begin{array}{l}\text { Valores encontrados } \\
\qquad(\mathrm{mg} / \mathrm{g})\end{array}$} & \multicolumn{2}{|c|}{$\begin{array}{l}\text { Coeficiente de } \\
\text { Variação (\%) }\end{array}$} & \multicolumn{2}{|c|}{ Média } & \multicolumn{2}{|c|}{$\begin{array}{l}\text { Porcentagem } \\
\text { Recuperação }\end{array}$} \\
\hline & Gluco & Condro & Gluco & Condro & Gluco & Condro & Gluco & Condro \\
\hline 1 (Gluco $400 \mathrm{mg} / \mathrm{g}$ & 406,1 & 475,5 & & & & & & \\
\hline Condro 480 mg/g) & $\begin{array}{l}393,2 \\
395,3\end{array}$ & $\begin{array}{l}488,3 \\
482,2\end{array}$ & 1,7 & 1,3 & 398,2 & 482,0 & 99,6 & 100,4 \\
\hline $\begin{array}{l}2(\text { Gluco } 500 \mathrm{mg} / \mathrm{g} \\
\text { Condro } 400 \mathrm{mg} / \mathrm{g} \text { ) }\end{array}$ & $\begin{array}{l}499,0 \\
510,7 \\
512,4\end{array}$ & $\begin{array}{l}408,9 \\
410,0 \\
397,5\end{array}$ & 1,4 & 1,7 & 507,3 & 405,5 & 101,5 & 101,4 \\
\hline $\begin{array}{l}3 \text { (Gluco } 400 \mathrm{mg} / \mathrm{g} \\
\text { Condro } 320 \mathrm{mg} / \mathrm{g} \text { ) }\end{array}$ & $\begin{array}{l}390,7 \\
388,8 \\
396,5\end{array}$ & $\begin{array}{l}322,3 \\
325,3 \\
319,6\end{array}$ & 1,0 & 0,9 & 392,0 & 322,4 & 98,0 & 100,7 \\
\hline $\begin{array}{l}4 \text { (Gluco } 600 \mathrm{mg} / \mathrm{g} \\
\text { Condro } 320 \mathrm{mg} / \mathrm{g} \text { ) }\end{array}$ & $\begin{array}{l}606,0 \\
595,2 \\
593,7\end{array}$ & $\begin{array}{l}316,0 \\
329,8 \\
324,9\end{array}$ & 1,1 & 2,2 & 598,3 & 323,6 & 99,7 & 101,1 \\
\hline
\end{tabular}

Tabela 4. Resultados de previsão para amostras de medicamentos obtidas no mercado utilizando-se o método desenvolvido e validado

\begin{tabular}{lcccccccc}
\hline Medicamentos & \multicolumn{2}{c}{$\begin{array}{c}\text { Concentração } \\
\text { declarada }\end{array}$} & \multicolumn{2}{c}{$\begin{array}{c}\text { Concentração } \\
\text { encontrada }\end{array}$} & \multicolumn{2}{c}{ Erro (\%) } & \multicolumn{2}{c}{$\begin{array}{c}\text { do valor } \\
\text { declarado }\end{array}$} \\
& Gluco & Condro & Gluco & Condro & Gluco & Condro & Gluco & Condro \\
\hline Manipulado 1 & 500,0 & 400,0 & 523,4 & 389,2 & $-4,7$ & 2,7 & 104,7 & 97,3 \\
Manipulado 2 & 500,0 & 400,0 & 489,1 & 422,8 & 2,2 & $-5,7$ & 97,8 & 105,7 \\
Manipulado 3 & 500,0 & 400,0 & 490,4 & 412,7 & 1,9 & 3,2 & 98,1 & 103,2 \\
\hline
\end{tabular}

diária foram satisfatórios, tendo um coeficiente de variação inferior ao valor máximo permitido para este teste, que é de $5 \% .{ }^{15}$

\section{Exatidão}

A exatidão do modelo multivariado foi avaliada por ensaio de recuperação em que quantidades conhecidas dos fármacos foram adicionadas ao excipiente estearato de magnésio. De acordo com a legislação vigente, ${ }^{15}$ os valores de recuperação devem estar entre 98 e $102 \%$ com relação ao valor esperado. Os resultados do ensaio de recuperação são apresentados na Tabela 3.

Os valores encontrados estão dentro do intervalo de 98 a 101,5\%, demonstrando resultado satisfatório para o ensaio de exatidão.

\section{Análise de medicamentos}

O modelo desenvolvido e validado foi utilizado para a determinação de 3 formulações obtidas em farmácias de manipulação.

Os resultados das análises destes medicamentos estão representados na Tabela 4.

O método desenvolvido teve boa capacidade de previsão para os medicamentos manipulados, apresentando como porcentagem do valor declarado 104,$7 ; 98,1$ e 97,8\% para a glucosamina e 97,3; 103,2 e $105,7 \%$ para a condroitina. Tais valores encontram-se dentro do intervalo de valores aceito para a análise de medicamentos, conforme monografias farmacopeicas. 


\section{CONCLUSÃO}

A espectroscopia no infravermelho, com técnica de reflectância difusa e regressão multivariada, apresentou-se adequada para determinação simultânea de glucosamina sulfato e condroitina sulfato em formulações farmacêuticas (cápsula).

$\mathrm{O}$ modelo de melhor capacidade preditiva foi aquele que considerou os espectros fragmentados das misturas, em que regiões de ruído foram previamente eliminadas e regiões de boa reprodução de bandas, observadas nos espectros das substâncias puras, foram consideradas.

Informações espectrais contidas na região do infravermelho próximo mostraram-se mais relevantes para o desenvolvimento do método, uma vez que o modelo de melhor desempenho considerou 910 números de onda na região do infravermelho próximo e apenas 156 números de onda no infravermelho médio.

O pré-processamento por correção multiplicativa de sinal (MSC) se apresentou útil para melhorar a capacidade preditiva do método, principalmente em razão de permitir a correção de problemas particularmente significativos nas técnicas fundamentadas em medidas de refletância.

$\mathrm{O}$ método mostrou-se preciso nos ensaios de repetitividade e precisão intermediária, exato e seletivo dentro do intervalo estabelecido de 400 a $600 \mathrm{mg} / \mathrm{g}$ para a glucosamina e 320 a $480 \mathrm{mg} / \mathrm{g}$ para a condroitina, com margens de erro e variação dentro das especificações apresentadas em normas nacionais e internacionais.

Este método, uma vez desenvolvido e validado, mostra-se promissor para utilização na rotina de produção já que as análises se tornam bastante rápidas, de baixo custo e praticamente sem produção de resíduos que causariam impacto ambiental.

\section{REFERENCIAS}

1. Mörgelin, M.; Paulsson, M.; Hardingham, T. E.; Heinegard, D.; Engel, J.; Biochem. J. 1988, 253, 175.

2. Coimbra, I. B.; Pastor, E. H.; D’Andrea, J. M. G.; Puccinelli, M. L. C.; Fuller, R.; Cavalcanti, F. S.; Maciel, F. M. B.; Honda, E.; Rev. Bras. Reumatol. 2002, 42, 371.

3. Correr, C. J.; Cordeiro, G.; Gasparetto, J.; Peralta-Zamora, P.; Pontarolo, R.; Acta Farm. Bonaerense. 2005, 24, 416.

4. Markopoulou, C. K.; Malliou, E. T.; Koundourellis, J. E.; J. Pharm. Biomed. Anal. 2005, 37, 249.

5. Dou, Y.; Mi, H.; Zhao, L.; Ren, Y.; Anal. Biochem. 2006, 17,174.

6. Coimbra, M. A.; Goncalves, F.; Barros, A. S.; Delgadillo, I. J.; Food Chem. 2002, 50, 3405.

7. Fuller, M. P. E.; Griffiths, P. R.; Anal. Chem. 1978, 50, 1906.

8. Reich, G.; Adv. Drug Delivery Rev. 2005, 57, 1109.

9. Özdemir, D.; Öztürk, B.; Turk. J. Chem. 2004, 28, 497.

10. Olinger, J. M.; Griffiths, P. R.; Appl. Spectrosc. 1993, 47, 687.

11. Skoog, D. A.; Holler, F. J.; Nieman, T. A.; Princípios de análise instrumental, $5^{\mathrm{a}}$ ed., Bookman: Porto Alegre, 2002.

12. Krivácsy, Z.; Hlavay, J.; J. Mol. Struct. 1995, 349, 289.

13. Milller, C. E.; Chemom. Intell. Lab. Syst. 1995, 30, 11.

14. Braga, J. W. B.; Poppi, R. J.; Quim. Nova 2004, 27, 1004.

15. Brasil. Resolução-RE n ${ }^{\circ} 899$ de 29 de maio de 2003; Diário Oficial da União, de 02 jun 2003, Brasília, 2003

16. Cerqueira, E. O.; Poppi, R. J.; Kubota, L.T.; Quim. Nova 2000, 23, 690. 